\title{
Assessment of hazard distribution within network systems with resistance feature
}

\author{
B. Jokšas ${ }^{1}$, I. Žutautaite-Šeputiene $\dot{e}^{1,2}$, J. Augutis ${ }^{1,2}$ \& E. Ušpuras ${ }^{1}$ \\ ${ }^{1}$ Lithuanian Energy Institute, Kaunas, Lithuania \\ ${ }^{2}$ Vytautas Magnus University, Kaunas, Lithuania
}

\begin{abstract}
In this paper a developed mathematical model of hazard distribution within a network system with a resistance feature is presented. The network system is described by graph theory. Hazards arising can be transmitted to others nodes which have connections with the infected node. Intensity of hazard transmission is described by channel bandwidth between the nodes of the network system. Each node has the ability to resist incoming hazards (to reduce it to safe level) in a network system. This ability is called resistance of the node. Resistance (or immunity) is regarded as random variables (or a random process) and Beta distribution is an appropriate probabilistic model of the node resistance.

The main purpose of this research work is developing a mathematical model that allows us to make forecast of how many cycles are necessary on average to eliminate hazards or to reduce to a safe level; how long (how many average cycles) system can work normally (corresponding to safety requirements) under the influence of hazard. Developed mathematical model of hazard distribution in a network system with resistance feature could be used in the assessment of operation safety of emergency systems that are provided to operate in extreme conditions during an accident as well.
\end{abstract}

Keywords: hazard distribution, resistance, Bayesian approach.

\section{Introduction}

Not always is it possible to perform reliability analysis for devices separately. Because, usually, devices don't operate separately, so its influence on each other must be considered. Combinations of devices, humans etc., structure network system (i.e. nodes and network lines or channels that represent interaction 
between components). Hazard in the risk analysis is defined as a feature or characteristic of material, technological process, information, human activities or other phenomena that specifies a potential possibility of danger for humans, nature, buildings, equipment and others. Illustrations of a hazard could be poisonous chemical substances kept in stock, or open sources of radiation, etc. The hazard is transmitted from one node to others trough the network channels. On the other hand, each node has the ability to resist the incoming hazard (to block its transmission or to diminish it). This property of node (i.e. software/ device/human) is called resistance. Node resistance can be created by the security systems. For instance, electricity distribution within networks with sophisticated automatic-protection against short circuits, network congestion, lines cracks and others. The purpose of this equipment is to localize failure (hazard) and to prevent it from spreading deeper into the network. It is of high importance for the future of electric distribution and transmission networks. It is similar to the smart grid. The systems for the assessment of reliability of hazardous materials transportation are going to be implemented in many countries. The detailed accounting of hazardous cargo routes and management are installed in these systems. Such system helps to divert hazardous cargos into more reliable routes in cases of traffic jams or other vehicle accidents, i.e., this system operates as a protection or resistance. These and similar systems require a mathematical model of hazard distribution within the network systems. The developed model helps to forecast the dependability level of the operating network system: to forecast how many cycles (it could be deterministic value for example: hours, days, etc.) are necessary to eliminate hazard or to reduce to safe level; how long (how many cycles) the system can work normally under the influence of a hazard.

\section{Model of distribution within network system}

First of all let us define several concepts that will be used in this paper:

A number of network nodes. The number of network nodes is marked as $N$.

Additive hazard. It is a sort of hazard, when hazards in the nodes of the network can be added to or a part of hazard moved to the other nodes (marked as $H)$.

Flow intensity $q_{i j}: q_{i j} \geq 0$ - coefficient of flow intensity in the network lines or channel; it marks the part of the hazard in the node $i$ that will be transmitted to the node $j$ [1]. The intensity of the flow to the node $j$ and the intensity of the flow from the node $j$ are defined respectively

$$
\breve{q}_{j}=\sum_{\substack{i=1 \\ i \neq j}}^{N} q_{i j}, \widehat{q}_{j}=\sum_{\substack{k=1 \\ k \neq j}}^{N} q_{j k} .
$$

Hazard transfer cycle. Hazard transfer within the network from one node to the other is regarded as one hazard transfer cycle.

Network node resistance. Coefficient marked as $I_{(j)}(\cdot)$. It marks which part of the hazard is stopped, before getting in the node $j\left(0 \leq I_{(j)} \leq 1\right.$, i.e. percentage). "Observed" value of node $j$ resistance could be obtained 


$$
I_{j(k)}=\frac{\breve{q}_{j(k)}-P_{j(k)}}{\breve{q}_{j(k)}},
$$

$k=1,2, \ldots$, here $k$-number of cycles, $\breve{q}_{j(k)}$ - the flow of hazard to the node $j$ in the $k^{\text {th }}$ cycle, $P_{j(k)}$ - amount of hazard that gets into the node $j$ during the $k^{\text {th }}$ cycle.

Mathematical model of hazard distribution within network system with deterministic resistances of nodes was proposed in Augutis \& Ušpuras (2006) research work [2]. Hazard in nodes after $k+1$ cycles

$$
\vec{H}(k+1)=\vec{H}(k) \cdot \widetilde{Q}+\vec{H}(0),
$$

$\widetilde{Q}$ - network flow matrix, with respect of resistances $I_{(j)}, j=1, \ldots, N$, of nodes, is defined as

$$
\widetilde{Q}=\left(\begin{array}{cccc}
q_{11} & q_{12}\left(1-I_{(2)}\right) & \cdots & q_{1 N}\left(1-I_{(N)}\right) \\
q_{21}\left(1-I_{(1)}\right) & q_{22} & \cdots & q_{2 N}\left(1-I_{(N)}\right) \\
\vdots & \vdots & & \vdots \\
q_{N 1}\left(1-I_{(1)}\right) & q_{N 2}\left(1-I_{(2)}\right) & \cdots & q_{N N}
\end{array}\right),
$$

$q_{i j}:\left\{0 \leq q_{i j} \leq 1, q_{\mathrm{il}}+\ldots+q_{\mathrm{iN}}=1\right\}$ marks the part of the hazard in the node $i$ that will be transmitted to the node $j$.

Hazard distribution within network system with resistance of nodes $\left(I_{(j)}{ }^{-}\right.$ nodes' resistance value into the node $j ; q_{i j}$ part of the hazard in the node $i$ that will be transmitted to the node $j$ ) is presented in Figure 1.

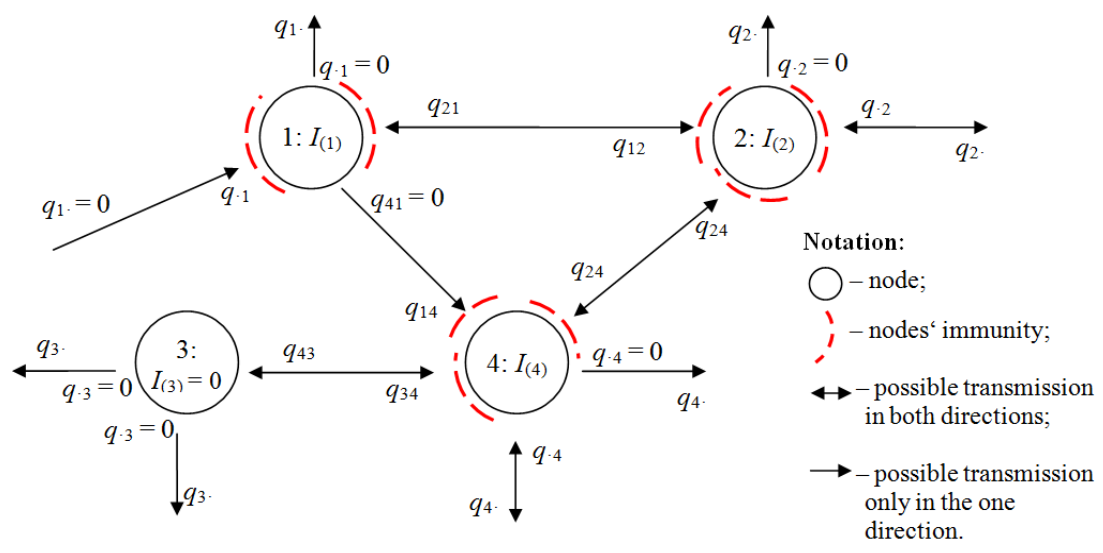

Figure 1: Fragment of network system.

Performing reliability analysis of real system true values of system nodes resistances are unknown. In this case resistance of node is assumed as random variable. In this paper developed model (presented in [1]) of hazard distribution is extended by two cases of unknown random resistance: 
- Invariable resistance (but unknown value of it) that is assumed as random variable. In this case it is important to obtain estimate of node resistance that is necessary for the forecast of the hazard level in node (in all network system as well). For instance, forecast how many cycles are necessary to reduce hazard to safe level or to eliminate, how long (how many cycles) system can work normally under the influence of hazard - to make decision in what moment supporting system should be switched on (i.e. alert generation).

- Inconstant resistance (increasing or decreasing) with known trend of it. For instance, self-learning system has increasing resistance. In this case concerned forecast - how many cycles are necessary to eliminate hazard or to reduce to safe (or acceptable) level. In the period of ageing system has decreasing resistance. In each cycle it is essential to have forecast how long (how many cycles) system can work normally under the influence of hazard. For instance, running device is degrading because of the influence of hazard, but it still has ability to diminish hazard. If the maintenance of device isn't costly it is expedient to use the device as long as it satisfies safety requirements before switching on of expensive supporting security system.

\section{Mathematical model of node resistance}

At first we need to choose model selection for node resistance. Resistance probabilistic distribution must have the following properties:

- Case 1 (invariable resistance). Value of resistance belongs to interval $[0,1]$.

- Case 2 (inconstant resistance). Value of resistance belongs to interval $[0,1]$. The mean of resistance satisfy equality

$$
\mathrm{E} I_{(j)}(k)=f_{(j)}\left(k, \theta_{1}, \ldots, \theta_{s_{(j)}}\right),
$$

$f_{(j)}(k, \cdot)$ - known function (i.e. linear, exponential and so on trend function) that depends on $k$-cycle number and parameters $\theta_{i}, i=1, \ldots, s_{(j)}, j=1, \ldots, N, N-$ number of nodes. Note in this paper analyzed case when resistance trend function depends on cycle number only. But in common it can be dependent on more than one factor, i.e. in this work all proposed models will be suitable as well. Variance of resistance is small, when the mean of resistance is near 0 or 1 (see Figure 2). In both cases (of invariable and inconstant resistance) Beta distribution is acceptable model for resistance.

Suppose it is possible to do measurements or observations of node resistance. For instance, hazard - high temperature, resistance measurement - how many degreases temperature was lowered by the node. In this case estimates of parameters in resistance model could be obtained (and updated) by algorithm that is based on BA (or modified application of BA [3]). Note "observed" values of node $j$ resistance are $I_{(j) k}: 0 \leq I_{(j) k} \leq 1, k=1,2, \ldots,(k-$ number of cycle $)$, $j=1,2, \ldots, N(N-$ number of nodes $)$. 


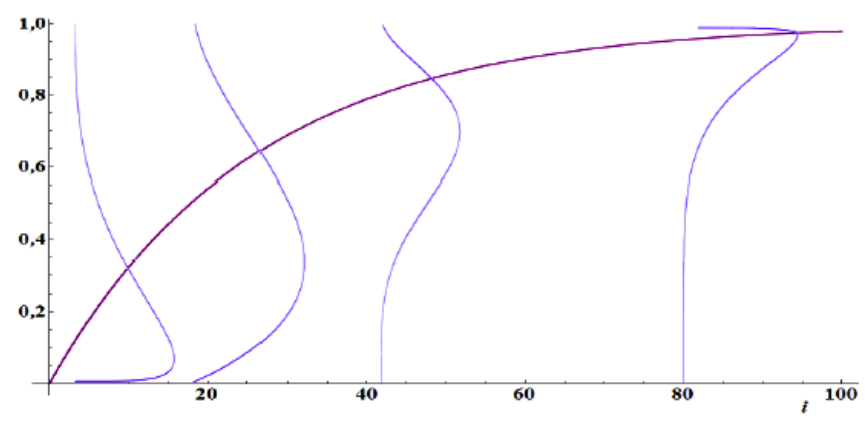

- exponentially increasing mean of resistance.

Figure 2: Transformation of density function.

Case 1 (invariable resistance). Resistance $I_{(j)}$ of node $j$ is approximated by Beta distribution $B e\left(\alpha_{(j)}, \beta_{(j)}\right)$. BA is applied to obtain estimates of parameters $\alpha_{(j)}$ and $\beta_{(j)}$. Posterior density function of its is

$$
\varphi\left(x, y \mid I_{(j) 1}, \ldots, I_{(j) k}\right)=\frac{\varphi(x, y) \prod_{i=1}^{k} \frac{1}{B(x, y)}\left(I_{(j) i}\right)^{x-1}\left(1-I_{(j) i}\right)^{y-1}}{\int_{0}^{\infty} \int_{0}^{\infty} \varphi(u, v) \prod_{i=1}^{k} \frac{1}{B(u, v)}\left(I_{(j) i}\right)^{u-1}\left(1-I_{(j) i}\right)^{v-1} \mathrm{~d} u \mathrm{~d} v},
$$

$\varphi(x, y)-$ prior density function of parameters $\alpha_{(j)}$ and $\beta_{(j)}$. BA point estimates of parameters $\alpha_{(j)}$ and $\beta_{(j)}$ are

$$
\begin{gathered}
\hat{\alpha}_{(j) k}=\int_{0}^{\infty} \int_{0}^{\infty} x \cdot \varphi\left(x, y \mid I_{(j) 1}, \ldots, I_{(j) k}\right) \mathrm{d} x \mathrm{~d} y, \\
\hat{\beta}_{(j) k}=\int_{0}^{\infty} \int_{0}^{\infty} y \cdot \varphi\left(x, y \mid I_{(j) 1}, \ldots, I_{(j) k}\right) \mathrm{d} x \mathrm{~d} y, k=2, \ldots, j=1, \ldots, N .
\end{gathered}
$$

Resistance point estimate is obtained as mean of updated Beta distribution

with variance

$$
\hat{I}_{(j)}=\mathrm{E} I_{(j)}=\frac{\hat{\alpha}_{(j) k}}{\hat{\alpha}_{(j) k}+\hat{\beta}_{(j) k}},
$$

$$
\operatorname{Var} I_{(j)}=\frac{\hat{\alpha}_{(j) k} \hat{\beta}_{(j) k}}{\left(\hat{\alpha}_{(j) k}+\hat{\beta}_{(j) k}\right)^{2}\left(\hat{\alpha}_{(j) k}+\hat{\beta}_{(j) k}+1\right)} .
$$

Case 2 (inconstant resistance). Resistance $I_{(j)}(k)$ of node $j$ is approximated by Beta distribution $\operatorname{Be}\left(\alpha_{(j)}(k), \beta_{(j)}(k)\right), k$ - number of cycle, and trend function (mean) of resistance is known

$$
\mathrm{E} I_{(j)}(k)=f_{(j)}\left(k, \theta_{1}, \ldots, \theta_{s_{(j)}}\right),
$$

it depends on number of cycle $k$ and parameters $\theta_{l}$ (that are assumed as random variables with prior probability density functions $\left.p_{l}\left(x_{l}\right)\right), l=1, \ldots, s_{(j)}$, and variance

$$
\operatorname{Var} I_{(j)}(k)=g_{(j)}\left(k, \kappa_{1}, \ldots, \kappa_{h_{(j)}}\right) \cdot
$$


In this case variable is inconstant, expressed as known function that depends on cycle number $k$ and parameters $\kappa_{i}$ (that are assumed as random variables with prior probability density functions $\left.\left.f_{i}\left(z_{i}\right)\right), i=1, \ldots, h_{(j)}\right)$. In general, variance could be unknown and assumed as random variable (its point estimate is obtained by BA as well) or the variance could be known constant. Note, if there is no prior information about variance of resistance, it could be used such concatenation between parameters $\alpha_{(j)}(k)$ and $\beta_{(j)}(k)$ of Beta distribution.

Note, if resistance is distributed by Beta distribution and mean of this distribution is varied exponentially, this variance of resistance is dependent on mean of the Beta distribution. When $\mathrm{E} I_{(j)} \rightarrow 0$ or $\mathrm{E} I_{(j)} \rightarrow 1$ then $\operatorname{Var} I_{(j)} \rightarrow 0$. In this paper, we analyzed the case when dependence between parameters of Beta distribution implements these assumptions, i.e., parameters satisfy following equality

$$
\beta_{(j)}\left(k, \theta_{1}, \ldots, \theta_{s_{(j)}}\right)=\frac{1}{\alpha_{(j)}\left(k, \theta_{1}, \ldots, \theta_{s_{(j)}}\right)} .
$$

This case of Beta distribution with concatenation (eqn. (13)) between parameters was analyzed in more details, because it requires less prior information than others (information about the variance of resistance is not necessary). In this case (of resistance as a random process) BA modified application is used to update point estimates of the random parameters of considered model. Parameter estimates $\theta_{l}, l=1, \ldots, s_{(j)}$ are obtained as expected value of posterior probability density function

$$
\hat{\theta}_{l}=\int_{R_{\theta_{l}}} x_{l} \varphi\left(\cdot \mid I_{(j) 1}, \ldots, I_{(j) n}\right) \mathrm{d} x_{l},
$$

$\varphi\left(\cdot \mid I_{(j) 1}, \ldots, I_{(j) n}\right)$ - posterior probability density function;

$R_{\theta_{l}}$-range (set of all possible values) of parameter $\theta_{l}, l=1, \ldots, s$.

Estimates of the parameters of resistance distribution (Beta distribution) $\hat{\alpha}_{(j)}\left(k, \hat{\theta}_{1}, \ldots, \hat{\theta}_{s_{(j)}}, \hat{\kappa}_{1}, \ldots, \hat{\kappa}_{h_{(j)}}\right), \hat{\beta}_{(j)}\left(k, \hat{\theta}_{1}, \ldots, \hat{\theta}_{s_{(j)}}, \hat{\kappa}_{1}, \ldots, \hat{\kappa}_{h_{(j)}}\right)$, that satisfy eqn. (11) and (12),

$$
\hat{\kappa}_{l}=\int_{R_{\theta_{l}}} z_{l} \varphi\left(\cdot \mid I_{(j) 1}, \ldots, I_{(j) n}\right) \mathrm{d} z_{l} .
$$

When concatenation between parameters $\alpha_{(j)}(k)$ and $\beta_{(j)}(k)$ is defined by eqn. (13) estimate of parameter $\alpha_{(j)}(k)$ is

$$
\hat{\alpha}_{(j)}\left(k, \hat{\theta}_{1}, \ldots, \hat{\theta}_{s_{(j)}}\right),
$$

$j=1, \ldots, N(N-$ number of nodes $), k$ - number of cycle.

The value of resistance of most systems can be equal to zero in general. If the resistance of system has possibility (or option) of self-learning, resistance reaches a value close to one. This value can be achieved in shorter or longer time, it depends on the specific conditions of the operation of the system. It could be possible to analyze changes of resistance dependence on the number of cycles or time. This dependence is determined according to prior information 
about similar systems, technical characteristic of systems protection (resistance). The exponential trend function of this dependence was analyzed in this work.

The case of exponentially increasing resistance was analyzed in more details. Assume that trend of node resistance is exponential

$$
\mathrm{E} I(k)=1-\mathrm{e}^{-a \cdot k}, a>0, k=1,2, \ldots,
$$

that depends on cycle number $k$ and random parameter $a$ (its prior probability density function $p(x))$. In this case is easy to prove that variance of resistance is bounded

$$
\operatorname{Var} I(k)<\left(1-\mathrm{e}^{-a \cdot k}\right) \mathrm{e}^{-a \cdot k}
$$

because $\operatorname{Var} I(k)<\mathrm{E} I(k)(1-\mathrm{E} I(k))$ (if resistance $I(k)$ follows Beta distribution), and the variance is bounded: $\max _{a, k} \operatorname{Var} I(k)<0.25$, i.e., the maximum value of variance is less than 0.25 (it is not difficult to prove).

Or in case when parameters of Beta distribution satisfy equality (13), posterior probability density function of parameter $a$ is

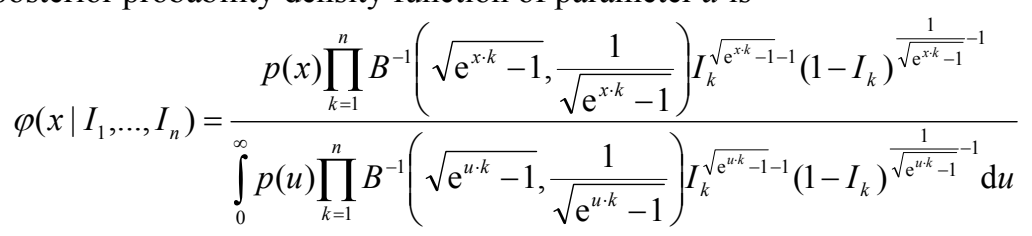

\section{Integration of resistance model into the hazard distribution model}

The model of hazard distribution within network system (defined by eqn (3)) is extended by incorporation of resistance probabilistic model into it. Actually, it is a challenging task. A fragment (three nodes connected in series, flow intensity coefficients of network lines are marked as $q_{i j}$ ), presented in Figure 3, of network system is analyzed in order to show the complexity of this problem.

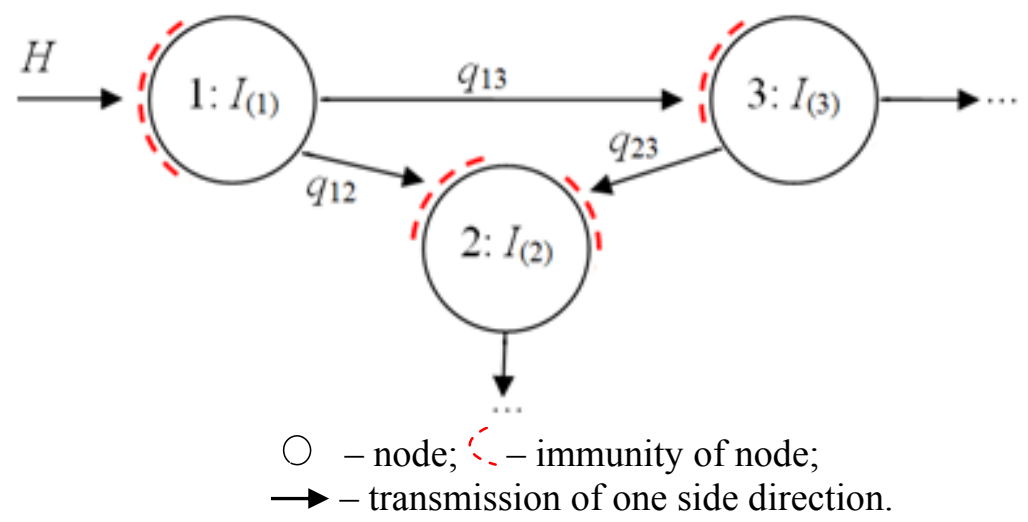

Figure 3: A fragment of network system (nodes' connection in series). 
In this case hazard distribution process is as follow

- the analyzed network system has point source of hazard (the amount of arisen hazard is $H$ );

- during the first cycle, hazard is transmitted to the first node: in the $1^{\text {st }}$ node the amount of hazard $H_{(1) 1}=H\left(1-I_{(1)}\right)$;

- during the second cycle, after the transmission hazard in the $2^{\text {nd }}$ node the amount of hazard $H_{(2) 2}=H_{(1) 1} q_{12}\left(1-I_{(2)}\right)=H q_{12}\left(1-I_{(1)}\right)\left(1-I_{(2)}\right)$, in the $3^{\text {rd }}$ node the amount of hazard $H_{(3) 2}=H_{(1) 1} q_{13}\left(1-I_{(3)}\right)=H q_{13}\left(1-I_{(1)}\right)\left(1-I_{(3)}\right)$;

- during the third cycle hazard form the $1^{\text {st }}$ and the $3^{\text {rd }}$ nodes is transmitted into the $2^{\text {nd }}$ node: the amount of hazard in the $2^{\text {nd }}$ node is $H_{(2) 3}=H_{(1) 2} q_{12}\left(1-I_{(2)}\right)$

$+H_{(3) 2} q_{23}\left(1-I_{(2)}\right)=H q_{12}\left(1-I_{(1)}\right)\left(1-I_{(2)}\right)+H q_{13} q_{23}\left(1-I_{(1)}\right)\left(1-I_{(3)}\right)\left(1-I_{(2)}\right)$;

- ...

Node resistance is assumed as random variable, hazard transmitted through any node became random variable as well. The resistance as a random variable follows Beta distribution, i.e., $I_{(j)} \sim B e\left(\alpha_{(j)}, \beta_{(j)}\right)$, opposite variable follows Beta distribution as well, i.e., $Z_{(j)}=1-I_{(j)} \sim B e\left(\beta_{(j)}, \alpha_{(j)}\right)$. In this way

- after the $1^{\text {st }}$ cycle: hazard in the $1^{\text {st }}$ node is defined as product of constant (initial hazard) and random variable which follows Beta distribution;

- after the $2^{\text {nd }}$ cycle: hazard in the $2^{\text {nd }}$ and in $3^{\text {rd }}$ nodes is defined as product of constant (i.e., product of initial hazard and flow intensity coefficient) and two random variables which follow Beta distribution;

- after the $3^{\text {rd }}$ cycle: hazard in the $2^{\text {nd }}$ node is defined as sum of two products of constant (i.e., product of initial hazard and flow intensity coefficients) and two (or three) random variables which follow Beta distribution;

This example shows that the incoming hazard is a random variable defined as a sum of products of random variables which follow Beta distribution. Our purpose is to obtain probability distribution of the hazard in nodes. This task was divided in two subtasks: the selection of probabilistic distribution of the product of Beta distributed random variables; the selection of probabilistic distribution of the sum of distributions which are used as approximations of the products of Beta distributed random variables.

Beta approximation. In probability theory it is known that the product of independent random variables distributed by Lognormal low as random variable follows Lognormal distribution as well, i.e. $X \sim \log N\left(\mu_{1}, \sigma_{1}{ }^{2}\right), Y \sim \log N\left(\mu_{2}, \sigma_{2}{ }^{2}\right)$, product $X \cdot Y \sim \log N\left(\mu_{1}+\mu_{2}, \sigma_{1}{ }^{2}+{\sigma_{2}}^{2}\right)$ [4]. Lognormal distribution was chosen for the approximation of Beta distribution because of it noticed useful property, i.e., this approximation provides simple (convenient for application) mathematical model of hazard distribution. Assume, that $X \sim B e(\alpha, \beta)$. Beta distribution is approximated by Lognormal distribution $\log N\left(\mu, \sigma^{2}\right)$. Parameters $\mu$ and $\sigma^{2}$ are computed by exactly matching the first and second central moments of random variable, i.e.

$$
\left\{\begin{array}{l}
\mathrm{E} X_{L o g N}=\mathrm{E} X_{\text {Beta }} \\
\operatorname{Var} X_{L o g N}=\operatorname{Var} X_{\text {Beta }}
\end{array}\right.
$$

The error of this approximation is of the order of the third moment. 
Lognormal sum approximation. The second subtask - analysis of the distribution of the sum of random variables which follow Lognormal distribution. The sum of Lognormal distributions (SLN) is well-known problem that no closed-form expression exists and it is difficult to evaluate numerically. Approximation of the SLN distribution by another Lognormal distribution, whose parameters are determined by moment (or cumulant) matching techniques, is widely used. The Fenton-Wilkinson [5] method is preferred in this research. Fenton-Wilkinson method gives approximation based on the first and second moments matching, i.e. $X_{1} \sim \log N\left(\mu_{1}, \sigma_{1}^{2}\right), X_{2} \sim \log N\left(\mu_{2}, \sigma_{2}^{2}\right)$, sum of these random variables $X=X_{1}+X_{2}$ is approximated by one lognormal distribution with parameters $\mu, \sigma^{2}$ that satisfy equations

$$
\left\{\begin{array}{l}
\mathrm{E} X_{1 \log N}+\mathrm{E} X_{2 \log N}=\mathrm{E} X_{\operatorname{LogN} N}, \\
\operatorname{Var} X_{1 \log N}+\operatorname{Var} X_{2 \log N}=\operatorname{Var} X_{\log N}
\end{array}\right.
$$

This kind of approximation enables one to develop a simple convenient mathematical model for hazard distribution. The error of this approximation is of the order of the third moment as well.

To conclude, the steps for the modeling of hazard distribution within the network system with resistance feature are:

1. during the $k^{\text {th }}$ cycle $\mathrm{BA}$ is used to obtain estimates of Beta distribution parameters $\hat{a}_{(j) k}$ and $\hat{\beta}_{(j) k}$, i.e., to obtain the updated distribution $\operatorname{Be}\left(\hat{\beta}_{(j) k}, \hat{a}_{(j) k}\right)$ of resistance of each node $j$;

2. it is defined distribution of opposite variable to resistance in each node $j$, i.e. $\left(1-I_{(j)}\right) \sim \operatorname{Be}\left(\hat{\beta}_{(j) k}, \hat{a}_{(j) k}\right)$;

3. in each node $j$ Beta distribution (of resistance) is approximated by Lognormal, i.e. $\operatorname{Be}\left(\hat{\beta}_{(j) k}, \hat{a}_{(j) k}\right) \Rightarrow \log N\left(\mu_{(j) k}, \sigma_{(j) k}^{2}\right)$, parameters $\mu_{(j) k}$ and $\sigma_{(j) k}^{2}$ are obtained as the result of (23) system of equations;

4. a) during the $k^{\text {th }}$ cycle hazard comes from nodes $r$ and $l$ to the node $d$, distribution of sum of random variables $\left(1-I_{(r)}\right)$ and $\left(1-I_{(l)}\right)$ approximated by one Lognormal distribution $\log N\left(\mu_{(d) k}=f\left(\mu_{(l) k}, \sigma_{(l) k}^{2}, \mu_{(r) k}, \sigma_{(r) k}^{2}\right)\right.$, $\left.\sigma_{(d) k}^{2}=g\left(\mu_{(l) k}, \sigma_{(l) k}^{2}, \mu_{(r) k}, \sigma_{(r) k}^{2}\right)\right)$ which parameters are obtained as the result of (21) system of equations;

4. b) distribution of product of random variables $\left(1-I_{(r)}\right)+\left(1-I_{(l)}\right)$ and (1$\left.I_{(j)}\right)$ is Lognormal $\log N\left(\mu_{(d) k}+\mu_{(j) k}, \sigma_{(d) k}^{2}+\sigma_{(j) k}^{2}\right)$.

Proposed probability distributions of nodes resistance are incorporated into the model of hazard distribution within the network system that is defined by eqn.(3).

In order to avoid approximation of Beta and Lognormal distributions mean value of hazard in each node could be used as it estimates. Mean of the sum (and the product) of independent random variables is easy computed as sum (or product) of means of analyzed random variables. 


\section{Results and conclusions}

The developed mathematical model of the hazard distribution within a network system with a resistance (assumed as a random variable or random process) feature is presented in this paper.

- Beta distribution is selected as more suitable for the modeling of node resistance as random variable (or random process with prior known trend function).

- The algorithm (based on Bayesian approach) for the estimation of parameters of resistance mathematical model is developed.

- Approximation of resistance Beta distribution by Lognormal distribution using Fenton-Wilkinson method was presented.

The developed mathematical model of hazard distribution within the network system with immunity feature could be used in development of emergency systems (for instance, accident localization system of nuclear power plant) to forecasts how long normal (corresponding to safety requirements) operation of the system is possible under extreme conditions during the accident.

\section{References}

[1] Augutis J., Ušpuras E. 2006. Monograph: Technology risk // Kaunas: Lithuanian Energy Institute, Vytautas Magnus University, pp. 30-55 ISBN 9986-492-89-0.

[2] Augutis, J., Krikstolaitis, R., Urbonas, R. \& Ušpuras, E. Hazard distribution and risk assessment in the network systems. Stochastic environmental research and risk assessment, 2006. Vol. 21, No. 1, pp. 51-61.

[3] Žutautaitė-Šeputienė I., Augutis J., Ušpuras E. Modelling of the node immunity change process in a network system // Risk analysis VI: sixth international conference on computer simulation risk analysis and hazard mitigation, Greece, May 5-7, 2008. Southampton, Boston: Wit Press, 2008. ISBN 978-1-84564-104-7, p. 357-365.

[4] Bareikis G., Šiaulys J., The product of independent random variables. VU press, 1998. pp. 27-34.

[5] Fenton L. F. The sum of lognormal probability distributions in scatter transmission systems, IRE Trans. Commun. Syst., 1960. vol. CS-8, pp. 5767.

[6] Wu J., Mehta N. \& Zhang J. A flexible lognormal sum approximation. Proceedings of IEEE Global Telecommunications Conference GLOBECOM 2005. Vol. 6. pp. 3413-3417. 